\section{Annals of \\ Chromatography and Separation Techniques}

\section{Article Information}

Received date: Sep 01, 2015

Accepted date: Sep 10, 2015

Published date: Sep 22, 2015

\section{${ }^{*}$ Corresponding author}

Ming-Fei Li, Beijing Key Laboratory of Lignocellulosic Chemistry, Beijing Forestry University, PR China, Tel/Fax: +8610 62336972; Email: limingfei@bjfu. edu.cn

Distributed under Creative Commons CC-BY 4.0

Keywords Fractionation; Biorefinery; Lignin; Bamboo

\section{Structural Characterization of Bamboo Lignin Isolated With Formic Acid and Alkaline Peroxide by Gel Permeation Chromatography and Pyrolysis Gas Chromatography Mass Spectrometry}

\author{
Xun $\mathrm{Li}^{1}$, Chang-Zhou Chen ${ }^{1}$ and Ming-Fei $\mathrm{Li}^{1 \star}$ \\ ${ }^{1}$ Beijing Key Laboratory of Lignocellulosic Chemistry, Beijing Forestry University, PR China
}

Abstract

Fractionation is an effective technology to maximize the utilization of lignocelluloses for the production of chemicals and materials. In this case, bamboo was subjected to a two-step fractionation process based on the concept of biorefinery: (a) formic acid treatment at boiling point under atmospheric pressure for $2 \mathrm{~h}$, and (b) posttreatment with alkaline hydrogen peroxide solution containing $1 \% \mathrm{NaOH}$ and $1 \% \mathrm{H}_{2} \mathrm{O}_{2}$ at $80^{\circ} \mathrm{C}$. The combination of formic acid delignification and alkaline hydrogen peroxide degradation achieved an effective removal of both lignin (delignification rate $94.9 \%$ ) and hemicelluloses (removal rate $87.4 \%$ ) from bamboo, producing celluloserich pulp, formic acid lignin and sugars. To investigate the structural modification of lignin during the fractionation process, the residual lignin in the treated samples was isolated and characterized with multiply techniques including gel permeation chromatography, pyrolysis gas chromatography mass spectrometry, Fourier-transform infrared spectroscopy, etc. The relative ratio of S/G was 1.63 for bamboo milled wood lignin (L1), whereas the lignin isolated from the formic acid treated cellulose-rich fraction (L2) presented a chromatograph similar to that of $L 1$ but had a lower $S / G$ ratio of 1.28. This indicated that a preferential removal of $S$ units during the formic acid fractionation process. In addition, alkaline hydrogen peroxide treatment resulted in more removal of $\mathrm{S}$ units, as indicated by a lower S/G ratio of 0.71 .

\section{Introduction}

In response to the current shortage of oil resource, much attention has been paid to lignocelluloses for the production of energy, chemicals and materials [1]. Bamboo is a feedstock used widely for paper, construction, furniture, as well as bio-ethanol, etc., mainly because it does not compete with food production, and it is fastest-growing and has a high bulk density. The value-added utilization of bamboo is associated with the complex structure of bamboo, which is resistant to processing thus efficient pretreatment method is required.

Organosolv treatment is a promising processing method implementing the concept of biorefinery through fractionation, which is based on the affinity of the components of lignocellulose towards different solvents. In general, organic solvents (alcohol, organic acid, ketone or ester) are utilized to dissolve lignin, accompanying cellulose in the solid residue. In addition, hemicelluloses can also be degraded and dissolved in liquid phase if water is added in the process. Many alternatives of the organosolv processes, such as AlCELL' (ethanol for lignin solvent), Lignol (ethanol for lignin solvent), Milox (formic acid for lignin solvent), Acetosolv (acetic acid for lignin solvent), Formacell (formic and acetic acids for lignin solvent), Organocell (methanol for lignin solvent), have been developed [2-5]. Among them, the process using formic acid as solvent can achieve an ideal delignification under mild conditions. The formic acid based processes have been utilized for many lignocelluloses including birch [6], sugarcane bagasse [7], corn (Zea mays L.) cob [8], etc. These treatments, which were conducted under atmosphere as compared to higher pressure in most fractionation process, resulted in the release of most of the lignin and hemicelluloses from lignocelluloses. Our major concern was to fractionate the main components from bamboo in an environmentally friendly and mild way. Bamboo has been subjected to formic acid process to release lignin to some extent in an earlier report [9]. Since there was some lignin in the solid residue after the formic acid treatment, a subsequent treatment was required. Therefore, the aim of the present study was to fractionate bamboo by a combination way and to investigate the structural changes of lignin during the process, since the behavior of lignin is of vital importance for the separation and utilization of lignocelluloses.

In this study, bamboo was delignified with formic acid followed by alkaline hydrogen peroxide, and the lignin in the treated samples was extracted and structurally characterized as compared to 
that from the original bamboo. The comprehensive characterization, including molecular weight, Fourier-Transform Infrared (FT-IR) spectroscopy, Pyrolysis gas chromatography mass spectrometry (Py-GC/MS), etc. is important not only for providing insight into the mechanism of lignin degradation but also for the processing of bamboo for pulp or biofuel, since the structure of lignin has a great effect on the processing technology.

\section{Materials and Methods}

Fractionation of bamboo by formic acid and alkaline hydrogen peroxide: Bamboo (B1) was subjected to formic acid delignification and alkaline hydrogen peroxide treatment to release lignin and hemicelluloses to obtain cellulosic pulp. Bamboo (300 g) was delignified with formic acid $(3000 \mathrm{~mL}, 88 \mathrm{wt} \%)$ at boiling point under atmosphere pressure for $2 \mathrm{~h}$. After delignification, the mixture was filtrated to obtain spent liquor and cellulosic pulp. The pulp was washed with formic acid and water followed by drying to obtain formic acid pulp (B2). After this, formic acid pulp was treated with alkaline hydrogen peroxide solution containing $1 \% \mathrm{NaOH}$ and $1 \%$ $\mathrm{H}_{2} \mathrm{O}_{2}$ at $80{ }^{\circ} \mathrm{C}$ with a liquor to solid ratio of $20(\mathrm{~mL} / \mathrm{g})$ for $1 \mathrm{~h}$. The treated pulp was washed with water and dried before use (B3). The experiments in the present study were performed in triplicates and the errors were reported.

Milled Wood Lignin (MWL) isolation: MWL was isolated from bamboo according to a previous report. Twenty grams of dewaxed bamboo was ball-milled with a planetary mill (FRITSCH pulverlsette, Germany). Then the bamboo powder was extracted with1, 4-dioxane/ water $(200 \mathrm{~mL}, 96 / 4, \mathrm{v} / \mathrm{v})$. After the evaporation of solution at $40{ }^{\circ} \mathrm{C}$ under reduced pressure, the lignin was purified by dissolution in acetic acid-water $(10 \mathrm{~mL}, 9 / 1, \mathrm{v} / \mathrm{v})$ followed by precipitation in 100 $\mathrm{mL}$ of water. The lignin fraction was dried and dissolved in $10 \mathrm{~mL}$ of 1,2-dichloroethane/ethanol, and precipitated in ether $(100 \mathrm{~mL})$. The precipitation was washed with ether and petroleum ether and dried. Then the lignin was subjected to acid hydrolysis as described in the part "Extraction of residual lignin" and named as L1.

Extraction of residual lignin: Extraction of residual lignin from the treated samples was conducted according to the procedure reported previously with minor modifications [10]. Cellulosic fraction (100 g) was refluxed with dioxane-water $(82 / 18, \mathrm{v} / \mathrm{v})$ solution $(1500 \mathrm{~mL})$ containing $0.1 \mathrm{M} \mathrm{HCl}$ for $2 \mathrm{~h}$ under nitrogen. Then the solid fraction was washed with dioxane/water $(82 / 18, \mathrm{v} / \mathrm{v})$ solution. The filtrate was evaporated to $50 \mathrm{~mL}$ under reduced pressure $(-0.1 \mathrm{MPa})$ at $40{ }^{\circ} \mathrm{C}$, and then the lignin was precipitated by the addition of water $(500 \mathrm{~mL})$. The precipitated lignin was then filtrated and washed with pentane to obtain purified lignin and named as L2 and L3, corresponding to the lignin extracted from cellulosic fractions obtained by formic acid delignification and alkaline hydrogen peroxide treatment, respectively.

Analysis methods: The chemical components of the samples were determined according to the National Renewable Energy Laboratory (NREL) method [11]. Molecular weight of the lignin was measured by a Gel Permeation Chromatography (GPC) system (Agilent Technologies) after acetylation. Elemental analysis was performed using a Vario El III analyzer. FT-IR spectra were recorded on a Thermo Scientific Nicolet iN10 FT-IR Microscope (Thermo Nicolet Corporation, Madison, WI, USA) equipped with liquid nitrogen cooled MCT detector.
Py-GC/MS analysis of the lignin was conducted with a multi-shot pyrolyzer (EGA/PY-3030D, Frontier Laboratories, Japan) combined with a GC-MS system (QP2010 Ultra, Shimadzu, Japan). The volatile pyrolysis products were separated by using an Ultra ALLOY+-5 $(30 \mathrm{~m} \times 0.25 \mathrm{~mm} \times 0.25 \mu \mathrm{m})$ columns. The fine powder $(0.25 \mathrm{mg})$ was loaded into a small platinum cup placed into a quartz tube and introduced into the pyrolysis chamber. The pyrolysis was conducted at $500^{\circ} \mathrm{C}$ for $10 \mathrm{~s}$. The pyrolysis interface was kept at $320^{\circ} \mathrm{C}$ and purged with helium to transfer the pyrolysis products to the GC column. The temperature of the chromatograph oven was programmed to rise from $50{ }^{\circ} \mathrm{C}(1 \mathrm{~min})$ to $280{ }^{\circ} \mathrm{C}$ at a rate of $3{ }^{\circ} \mathrm{C} / \mathrm{min}$, and to $300{ }^{\circ} \mathrm{C}$ at a rate of $30^{\circ} \mathrm{C} / \mathrm{min}$. The final temperature was held for $3 \mathrm{~min}$. The mass spectrometer was operated by electron impact ionization at 70 $\mathrm{eV}$. The temperatures of the detector and the GC/MS interface were $200{ }^{\circ} \mathrm{C}$ and $280{ }^{\circ} \mathrm{C}$, respectively. Identification of the products was conducted by comparison of their mass spectra with GC/MS library (National Institute of Standards and Technology 2005) and data from literature. Relative quantification was performed on the basis of the relative area of each lignin-derived compound divided by the total area of the chromatogram [12].

\section{Result and Discussion}

Effect of the formic acid delignification and alkaline hydrogen peroxide extraction: Bamboo was subjected to two environmentally friendly and mild fractionation processes to fractionate bamboo to obtain cellulose-rich pulp together with degraded lignin and hemicellulosic sugars. After the formic acid treatment and alkaline hydrogen peroxide fractionation process, the lignin and hemicelluloses in bamboo were notably removed. As it can be seen from the solid residue B2 (Table 1), the hemicelluloses content was $6.84 \%$ and the lignin content was $8.17 \%$. After a further fractionation with alkaline hydrogen peroxide, the hemicelluloses and lignin contents further decreased to $6.81 \%$ and $3.50 \%$, respectively. It was concluded that the combination of formic acid delignification and alkaline hydrogen peroxide degradation result in an effective removal of both lignin (delignification rate 94.9\%) and hemicelluloses (removal rate $87.4 \%$ ) from bamboo. The extraction efficiency was higher than the delignification with alcohols, which is usually conducted at higher temperatures (above $140{ }^{\circ} \mathrm{C}$ ) the delignification rate is less than $90 \%$ [13]. In addition, the extraction of hemicelluloses was conducted by diluted alkaline solution as compared to the conventional extraction with concentrated $\mathrm{NaOH}$, which was easy to treat the waste liquor after the fractionation process [14].

Table 1: Yield and chemical composition of the fractionated bamboo samples as compared to the original bamboo.

\begin{tabular}{|c|c|c|c|c|c|}
\hline \multirow{2}{*}{ Sample } & \multirow{2}{*}{ Yield $^{\mathrm{a}}(\%)$} & \multicolumn{4}{|c|}{ Chemical composition } \\
& & Cellulose & Hemicelluloses & Lignin & Extractives \\
\hline B1 & & $46.55 \pm 1.21$ & $19.76 \pm 0.23$ & $24.88 \pm 0.87$ & $3.95 \pm 0.02$ \\
\hline B2 & $46.00 \pm 1.10$ & $86.48 \pm 1.05$ & $6.84 \pm 0.56$ & $8.17 \pm 1.21$ & $0.51 \pm 0.15$ \\
\hline B3 & $36.51 \pm 0.52$ & $90.79 \pm 0.85$ & $6.81 \pm 1.20$ & $3.50 \pm 0.06$ & $0.32 \pm 0.03$ \\
\hline
\end{tabular}

${ }^{a}$ Based on the original bamboo; ${ }^{b}$ Based on the measured sample.

FT-IR spectra of the solid fractions were recorded for comparison (data not shown). For bamboo, the band at $3420 \mathrm{~cm}^{-1}$ is due to $\mathrm{O}-\mathrm{H}$ stretch and that at $2915 \mathrm{~cm}^{-1}$ is attributed to methyl, methylene and methane groups. Aromatic skeletal vibrations in lignin were observed at1596, 1506, and $1422 \mathrm{~cm}^{-1}$. The peak at 834 is from the C-H out of plane bending of $\mathrm{S}$ unit in lignin. These peaks of lignin noticeably diminished after the formic acid delignification as well as alkaline hydrogen peroxide fractionation. The peak at $1732 \mathrm{~cm}^{-1}$, increased 
Table 2: Weight-average (Mw) and number-average $(\mathrm{Mn})$ molecular weights and polydispersity (Mw/Mn) of bamboo lignin.

\begin{tabular}{|c|c|c|c|}
\hline Sample & L1 & L2 & L3 \\
\hline $\mathrm{Mw}(\mathrm{g} / \mathrm{mol})$ & $4650 \pm 100$ & $8490 \pm 250$ & $10340 \pm 120$ \\
\hline $\mathrm{Mn}(\mathrm{g} / \mathrm{mol})$ & $2100 \pm 150$ & $4390 \pm 220$ & $3200 \pm 220$ \\
\hline $\mathrm{Mw} / \mathrm{Mn}$ & 2.2 & 1.9 & 3.2 \\
\hline
\end{tabular}

Table 3: Elemental analysis and $\mathrm{C}_{9}$ formula of lignin.

\begin{tabular}{|c|c|c|c|c|c|}
\hline Sample & $\mathbf{C} \%$ & $\mathbf{H} \%$ & $\mathbf{O} \%$ & $\mathbf{O C H}_{3} \%$ & $\mathbf{C}_{9}$ \\
\hline L1 & $63.10 \pm 1.20$ & $5.67 \pm 2.01$ & $31.23 \pm 1.23$ & $17.74 \pm 0.29$ & $\mathrm{C}_{9} \mathrm{H}_{7.53} \mathrm{O}_{2.65}\left(\mathrm{OCH}_{3}\right)_{1.10}$ \\
\hline L2 & $64.58 \pm 0.06$ & $6.04 \pm 1.81$ & $29.38 \pm 1.54$ & $17.47 \pm 1.05$ & $\mathrm{C}_{9} \mathrm{H}_{8.06} \mathrm{O}_{2.38}\left(\mathrm{OCH}_{3}\right)_{1.05}$ \\
\hline L3 & $65.17 \pm 1.51$ & $7.10 \pm 1.26$ & $27.73 \pm 1.06$ & $18.14 \pm 0.87$ & $\mathrm{C}_{9} \mathrm{H}_{9.85} \mathrm{O}_{2.14}\left(\mathrm{OC}_{\mathrm{H} 3) 1} .09\right.$ \\
\hline
\end{tabular}

sharply in $\mathrm{B} 2$ due to esterification during the delignification process, but disappeared in $\mathrm{B} 3$ due to the hydrolysis of the ester bond in alkaline solution.

Structural characterization of lignin: As seen from Table 2, the lignin extracted from formic acid delignified bamboo had a higher weight average molecular weight $(8490 \mathrm{~g} / \mathrm{mol})$ as compared to the milled wood lignin from bamboo $(4650 \mathrm{~g} / \mathrm{mol})$. After a further extraction, the residual lignin in the bamboo showed a much higher weight average molecular weight $(10340 \mathrm{~g} / \mathrm{mol})$. This suggested the preferential release of lignin with low molecular weight during the fractionation process. The results were in well agreement with the previous report that the degradation and dissolution of lignin in the formic acid pulping process, in which the dissolved lignin showed lower molecular weight as compared to MWL [9].

The data from the elemental analysis and methoxy group content as well as the calculated C9 formula are listed in Table 3.

Table 4: Composition of the compounds released after Py-GC-MS of the different isolated lignin (relative errors are below 3\%).

\begin{tabular}{|c|c|c|c|c|c|c|}
\hline \multirow{2}{*}{ No. } & \multirow{2}{*}{ Name } & \multirow{2}{*}{ Type } & \multirow{2}{*}{ Mw } & \multicolumn{3}{|c|}{ Content (\%) } \\
\cline { 5 - 7 } & & L1 & L2 & L3 \\
\hline 1 & Phenol & 94 & 2.0 & 5.8 & 7.0 \\
\hline 2 & 4-Methylphenol & H & 108 & 2.9 & 3.4 & 5.1 \\
\hline 3 & Guaiacol & G & 124 & 5.7 & 7.7 & 10.2 \\
\hline 4 & 4-Ethylphenol & H & 122 & 1.9 & 0.7 & 1.9 \\
\hline 5 & 4-Methylguaiacol & G & 138 & 0.5 & 0.9 & 1.5 \\
\hline 6 & 4-Methylguaiacol & G & 138 & 8.6 & 10.0 & 13.7 \\
\hline 7 & 4-Vinylphenol & H/PCA & 120 & 21.1 & 16.0 & 13.9 \\
\hline 8 & 4-Ethylguaiacol & G & 152 & 1.8 & 2.5 & 3.4 \\
\hline 9 & 4-Vinylguaiacol & G/FA & 150 & 4.4 & 5.7 & 7.2 \\
\hline 10 & Syringol & S & 154 & 6.6 & 5.9 & 5.5 \\
\hline 11 & cis-Isoeugenol & G & 164 & 0.8 & 0.9 & 1.0 \\
\hline 12 & Vanillin & G & 152 & 2.1 & 1.8 & 2.1 \\
\hline 13 & trans-Isoeugenol & G & 164 & 0.5 & 0.7 & 0.9 \\
\hline 14 & 4-Methylsyringol & S & 168 & 10.7 & 11.0 & 9.2 \\
\hline 15 & p-Propylguaiacol & G & 166 & 1.2 & 1.0 & 1.2 \\
\hline 16 & Acetoguaiacone & G & 166 & 0.8 & 1.1 & 0.7 \\
\hline 17 & 4-Ethylsyringol & S & 182 & 1.0 & 0.9 & 2.9 \\
\hline 18 & Guaiacylacetone & G & 180 & 1.3 & 1.1 & 0 \\
\hline 19 & 4-Vinylsyringol & S & 180 & 3.4 & 2.3 & 2.8 \\
\hline 20 & Guaiacylacetone & G & 180 & 1.1 & 0.7 & 0.4 \\
\hline 21 & Guaiacyl Vinyl Ketone & G & 178 & 0.6 & 0.7 & 0.6 \\
\hline 22 & Acetoguaiacone & G & 166 & 0.7 & 1.1 & 2.2 \\
\hline 23 & 4-Allylsyringol & S & 194 & 1.1 & 1.0 & 0.4 \\
\hline 24 & cis-4-Propenylsyringol & S & 194 & 0.9 & 0.4 & 1.3 \\
\hline 25 & Syringaldehyde & S & 182 & 2.7 & 1.5 & 0 \\
\hline 26 & 4-Propinylsyringol & S & 192 & 4.2 & 3.8 & 2.3 \\
\hline 27 & 4-Propinylsyringol & S & 192 & 1.1 & 1.3 & 0.3 \\
\hline 28 & Acetosyringone & S & 196 & 4.3 & 2.1 & 1.5 \\
\hline 29 & Syringlacetone & S & 210 & 2.9 & 3.7 & 0.9 \\
\hline 30 & Propiosyringone & S & 210 & 1.2 & 1.6 & 0 \\
\hline 31 & trans-Sinapaldehyde & S & 208 & 1.9 & 2.8 & 0 \\
\hline & & & & & & \\
\hline
\end{tabular}

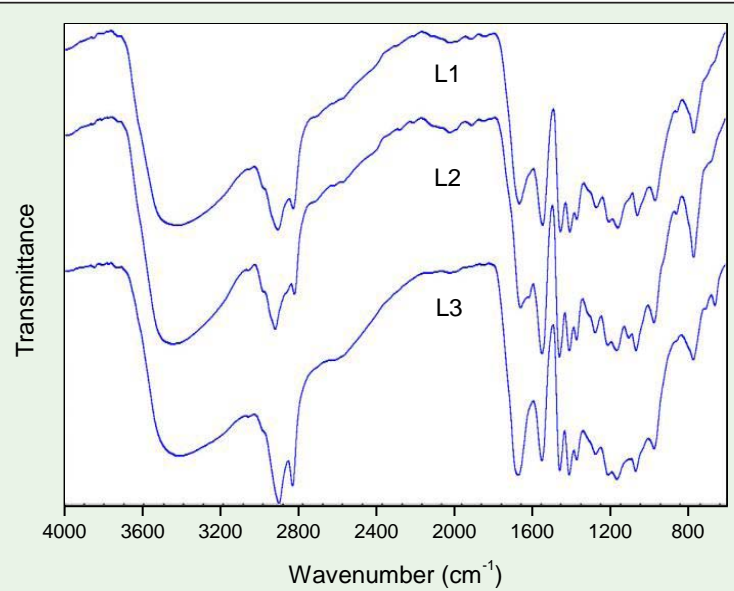

Figure 1: FT-IR spectra of lignin isolated from bamboo.

The residual lignin of bamboo exhibited an increase in $\mathrm{C}$ content but a decrease in $\mathrm{O}$ content when compared to the corresponding lignin from the original bamboo. This feature was in well agreement with the dissolution of non-condensed structure of lignin during the delignification process.

FT-IR spectra of the lignin isolated from the treated and untreated bamboo samples are presented in Figure 1. The variation of the spectra indicated that the functional groups of the lignin in bamboo changed to some extent after the treatments. The peak at $1719 \mathrm{~cm}^{-1}$ is assigned to the $\mathrm{C}=\mathrm{O}$ stretch in carbonyl, un-conjugated ketones, and in ester groups, whereas that at $1655 \mathrm{~cm}^{-1}$ corresponds to conjugated $p$-substituted aryl ketones. The band at $1510 \mathrm{~cm}^{-1}$ is assigned to the aromatic skeletal vibrations and that at $1460 \mathrm{~cm}^{-1}$ corresponds to the $\mathrm{C}-\mathrm{H}$ deformation, asymmetrical vibration in $\mathrm{CH}_{3}$ and $\mathrm{CH}_{2}$. The signal at $1420 \mathrm{~cm}^{-1}$ originates from the aromatic ring vibrations. The band at $1326 \mathrm{~cm}^{-1}$ indicates the $\mathrm{C}$ and $\mathrm{G}$ ring condensation ( $\mathrm{G}$ ring substituted in $\mathrm{C}-5$ position). The peak at $1224 \mathrm{~cm}^{-1}$ is assigned to $\mathrm{C}-\mathrm{C}, \mathrm{C}-\mathrm{O}$, and $\mathrm{C}=\mathrm{O}$ stretching ( $\mathrm{G}$ condensed $>\mathrm{G}$ etherified).
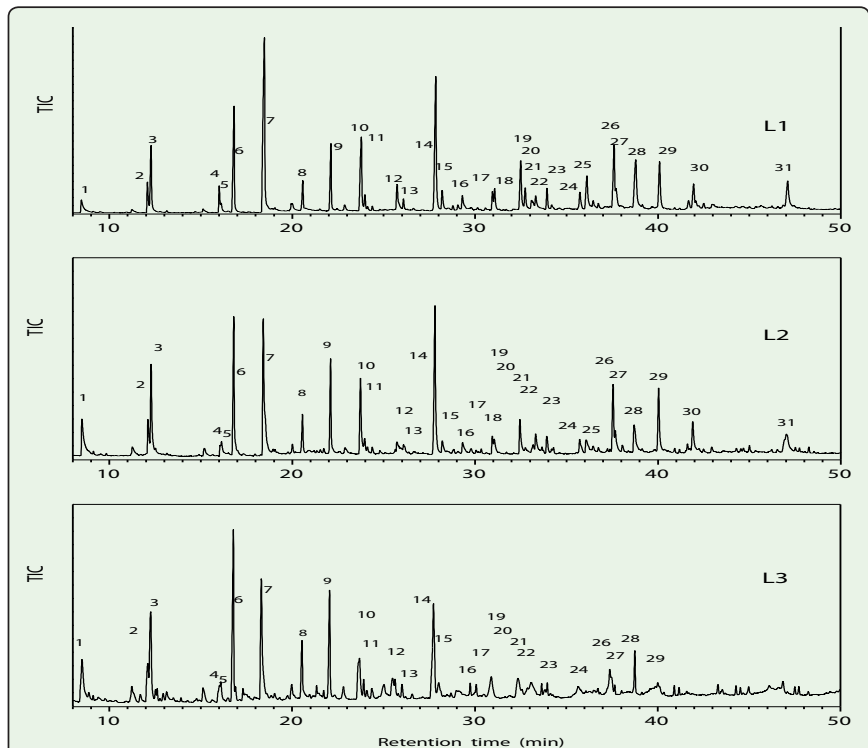

Figure 2: Py-GC/MS chromatograph of lignin isolated from bamboo. 
The intensities at $1127 \mathrm{~cm}^{-1}$ and $1034 \mathrm{~cm}^{-1}$ are due to the aromatic in plane $\mathrm{C}-\mathrm{H}$ bending and aromatic in-plane $\mathrm{C}-\mathrm{H}$ bending, respectively. The similarity of the signals between 1597 and $836 \mathrm{~cm}^{-1}$ suggested that the core structure of lignin did not change after the treatments. As compared the L1, the intensity at $1719 \mathrm{~cm}^{-1}$ increased slightly for L2 due to esterification, and it increased largely for L3 probably due to oxidization.

Py-GC/MS is a rapid and sensitive technique for analyzing the composition of lignin [15-17]. The chromatograms of lignin samples are illustrated in Figure 2 and the identities and relative abundances of the degraded compounds are shown in Table 4 . The chromatogram of bamboo MWL indicated that this lignin was a GSH type, in accordance with the literature [18]. The main compounds released were 4-vinylphenol (H/PCA), 4-methylsyringol (S), 4-methylguaiacol (G), guaiacol (G). The Py-GC/MS data indicated the relative ratio of the $\mathrm{S} / \mathrm{G}$ ratio was 1.63 for $\mathrm{L} 1$. The residual lignin isolated from the formic acid treated cellulose-rich fraction (L2) presented a chromatograph similar to that of bamboo MWL (L1) but had a low proportion of $\mathrm{S}$ units, resulting in a lower $\mathrm{S} / \mathrm{G}$ ratio of 1.28 . This indicated that a preferential removal of $S$ units in bamboo during the formic acid fractionation process. Alkaline hydrogen peroxide treatment also resulted in more removal of $S$ units, as indicated by a lower S/G ratio of 0.71 in L3. In short, formic acid and alkaline hydrogen peroxide treatments could liberate both $S$ and $G$ type lignin, in which more $S$ units were removed under the conditions studied.

\section{Conclusion}

Bamboo was fractionated by formic acid delignification and alkaline hydrogen peroxide post-treatment to remove lignin and hemicelluloses, producing cellulose-rich pulp. The structural modification of lignin was investigated by extracting lignin from bamboo and cellulose-rich residue and characterized by multiply technologies. Py-GC/MS analysis indicated that the relative ratio of $\mathrm{S} / \mathrm{G}$ was decreased from 1.28 in formic acid treated bamboo lignin to 0.71 in alkaline hydrogen peroxide treated bamboo lignin, as compare to 1.63 in the milled wood lignin of bamboo. This indicated that the preferential removal of $\mathrm{S}$ units during the formic acid fractionation process and that alkaline hydrogen peroxide treatment resulted in more removal of $\mathrm{S}$ units.

\section{Acknowledgement}

This work was supported by the Fundamental Research Funds for the Central Universities (No.YX2015-01).

\section{References}

1. Maity SK. Opportunities, recent trends and challenges of integrated biorefinery: Part I. Renew Sust Energ Rev. 2015; 43: 1427-1445.

2. Li M-F, Sun S-N, Xu F, Sun R-C. Organosolv fractionation of lignocelluloses for fuels, chemicals and materials: a biorefinery processing perspective. In: Biomass Conversion: The Interface of Biotechnology, Chemistry and Materials Science.C Baskar, S Baskar, RS Dhillon, Editors. Springer Berlin, Heidelberg. 2012; 341-379.
3. Pan X, Gilkes N, Kadla J, Pye K, Saka S, Gregg D, Ehara K. Bioconversion of hybrid poplar to ethanol and co-products using an organosolv fractionation process: optimization of process yields. Biotechnol Bioeng. 2006; 94: 851861.

4. de la Torre MJ, Moral A, Hernández MD, Cabeza E, Tijero A. Organosolv lignin for biofuel. Ind Crop Prod. 2013; 45: 58-63.

5. Fang W, Sixta $\mathrm{H}$. Advanced biorefinery based on the fractionation of biomass in $\gamma$-valerolactone and water. ChemSusChem. 2015; 8: 73-76.

6. Zhou S, Liu L, Wang B, Xu F, Sun RC. Microwave-enhanced extraction of lignin from birch in formic acid: structural characterization and antioxidant activity study. Process Biochem. 2012; 47: 1799-1806.

7. Zhao X, Liu D. Fractionating pretreatment of sugarcane bagasse by aqueous formic acid with direct recycle of spent liquor to increase cellulose digestibilitythe Formiline process. Bioresour Technol. 2012; 117: 25-32.

8. Zhang M, Qi W, Liu R, Su R, Wu S, He Z. Fractionating lignocellulose by formic acid: Characterization of major components. Biomass Bioenerg. 2010; 34: 525-532.

9. Li MF, Sun SN, Xu F, Sun RC. Formic acid based organosolv pulping of bamboo (Phyllostachys acuta): comparative characterization of the dissolved lignins with milled wood lignin. Chem Eng J. 2012; 179: 80-89.

10. Gellerstedt G, Pranda J, Lindfors EL. Structural and molecular-properties of residual birch kraft lignins. J Wood Chem Technol. 1994; 14: 467-482.

11. Sluiter A, Hames B, Ruiz R, Scarlata C, Sluiter J, Templeton D, Crocker D. Determination of structural carbohydrates and lignin in biomass. In: Laboratory Analytical Procedure. 2008.

12. Lopes FJF, Silvério FO, Baffa DCF, Loureiro ME, Barbosa MHP. Determination of sugarcane bagasse lignin $\mathrm{S} / \mathrm{G} / \mathrm{H}$ ratio by pyrolysis $\mathrm{GC} / \mathrm{MS}$. J Wood Chem Technol. 2011; 31: 309-323.

13. Yu H, You Y, Lei F, Liu Z, Zhang W, Jiang J. Comparative study of alkaline hydrogen peroxide and organosolv pretreatments of sugarcane bagasse to improve the overall sugar yield. Bioresour Technol. 2015; 187: 161-166.

14. Fares K, Renard C, Crepeau MJ, Thibault JF. Characterization of hemicelluloses of sugar beet roots grown in Morocco. Int J Food Sci Tech. 2004; 39: 303-309.

15. Kim J-Y, Hwang H, Park J, Oh S, Choi JW. Predicting structural change of lignin macromolecules before and after heat treatment using the pyrolysisGC/MS technique. J Anal Appl Pyrol. 2014; 110: 305-312.

16. Zhang M, Resende FL, Moutsoglou A. Catalytic fast pyrolysis of aspen lignin via Py-GC/MS. Fuel. 2014; 116: 358-369.

17. Zhao J, Xiuwen W, Hu J, Liu Q, Shen D, Xiao R. Thermal degradation of softwood lignin and hardwood lignin by TG-FTIR and Py-GC/MS. Polym Degrad Stabil. 2014; 108: 133-138.

18. del Río JC, Rencoret J, Prinsen P, Martínez ÁT, Ralph J, Gutiérrez A. Structural characterization of wheat straw lignin as revealed by analytical pyrolysis, 2D-NMR, and reductive cleavage methods. J Agric Food Chem. 2012; 60: 5922-5935. 